\title{
Rapid evolution of [WC] stars in the Magellanic Clouds ${ }^{\star}$
}

\begin{abstract}
Marcin Hajduk
Space Radio-Diagnostics Research Centre, University of Warmia and Mazury, ul.Oczapowskiego 2, 10-719 Olsztyn, Poland e-mail: marcin.hajduk@uwm.edu.pl

Received 30 March 2020 / Accepted 30 June 2020

ABSTRACT

We obtained new spectra of fourteen Magellanic Cloud planetary nebulae with the South African Large Telescope to determine heating rates of their central stars and to verify evolutionary models of post-asymptotic giant branch stars. We compared new spectra with observations made in previous years. Five planetary nebulae showed an increase in excitation over time. Four of their central stars exhibit [WC] features in their spectra, including three new detections. This raises the total number of [WC] central stars of PNe in the Magellanic Clouds to ten. We compared determined heating rates of the four [WC] central stars with the He-burning post-asymptotic giant branch evolutionary tracks and the remaining star with the H-burning tracks. Determined heating rates are consistent with the evolutionary models for both $\mathrm{H}$ and He-burning post-asymptotic giant branch stars. The central stars of the PNe that show the fastest increase of excitation are also the most luminous in the sample. This indicates that [WC] central stars in the Magellanic Clouds evolve faster than H-burning central stars, and they originate from more massive progenitors.
\end{abstract}

Key words. stars: AGB and post-AGB - planetary nebulae: general - stars: evolution - Magellanic Clouds

\section{Introduction}

Stars with initial masses in the range of $1-8 M_{\odot}$ lose most of their envelope on the asymptotic giant branch (AGB). During an early AGB phase, helium burning proceeds in a thin shell on the top of the carbon-oxygen core. When the helium shell reaches the hydrogen shell, the nuclear burning becomes unstable. A series of helium shell flashes punctuate quiescent hydrogen burning.

The large energy generation due to a helium flash induces a convective instability between the shells (Iben \& Renzini 1983; Herwig 2005). When the $\mathrm{H}$ shell becomes active again, part of the intershell material is dredged up to the surface.

The envelope of the star is rapidly expelled due to the intensive mass loss experienced during the AGB phase. This reduces the number of helium shell flashes. The mass ejected by AGB stars significantly contributes to the chemical evolution of galaxies and stellar systems (Karakas 2010).

After a star ejects most of its envelope, it terminates the AGB phase and its evolution accelerates. Heating rate increases from $\dot{T}_{*} \lesssim 0.1 \mathrm{kK} \mathrm{yr}^{-1}$ to $1 \mathrm{~K} \mathrm{yr}^{-1} \lesssim \dot{T}_{*} \lesssim 10 \mathrm{kK} \mathrm{yr}^{-1}$ (Miller Bertolami et al. 2019). The evolutionary timescale of post-AGB stars is a very strong function of their envelope mass and the nuclear burning rate. Post-AGB stars occupy a relatively narrow range in mass between 0.5 and $0.9 M_{\odot}$, but evolutionary timescales differ by a few orders of magnitude within this range.

Post-AGB stars cross the H-R diagram from low to high temperatures almost at constant luminosity, as long as the shell sources remain active (Paczyński 1970). Once the star is hot enough, the ionization front expands throughout the shell ejected during the AGB phase. The shell forms a planetary nebula (PN).

\footnotetext{
* The reduced spectra are only available at the CDS via anonymous ftp to cdsarc.u-strasbg.fr (130.79.128.5) or via http: //cdsarc.u-strasbg.fr/viz-bin/cat/J/A+A/642/A71
}

The excitation of the PN increases with the increasing temperature of the central star.

A central star of a PN may experience a final thermal pulse after it leaves AGB (Schoenberner 1979). A final thermal pulse can punctuate evolution of a post-AGB star immediately after it leaves the AGB (called AFTP), during its horizontal evolution, when H-burning shell is active (late thermal pulse - LTP), or during cooling (very late thermal pulse - VLTP; Koesterke 2001).

In the result of the VLTP, the pulse driven convecting zone eventually reaches out into the H-rich envelope (Herwig 2005). The LTP and AFTP lead to the diluted shell and significantly reduced $\mathrm{H}$ abundance. An AFTP can lead to both a considerable enrichment with carbon and oxygen and to the dilution of hydrogen. This depends on the envelope mass. The smaller envelope mass, the less hydrogen is left in the envelope (Blöcker 2001).

$\mathrm{H}$-deficient central stars constitute at least $30 \%$ of all the central stars in the Galaxy (Weidmann \& Gamen 2011). More than half of them are [WC] stars, which show emission line spectra typical for WR stars of carbon sequence (Acker \& Neiner 2003). Hydrogen-deficient central stars are believed to expose their intershell region in their atmospheres due to a late heliumshell flash (Werner \& Herwig 2006).

The evolution of post-AGB stars remains one of the most difficult topics in stellar evolution (Miller Bertolami et al. 2019). The models must rely on assumptions and approximations such as mixing length theory or stellar wind theory. Observational verification is necessary for the validation of the evolutionary models.

Stellar luminosities and temperatures have been traditionally used to study the evolution of post-AGB stars. However, postAGB stars occupy a narrow range in luminosities in the H-R diagram. Their distances are little known and the stars emit most of their energy in the ultraviolet range. Observational errors propagate large uncertainties in the determination of the luminosities. 
Table 1. Log of the PNe observations.

\begin{tabular}{lcccc}
\hline \hline Name & Exp. [s] & Date & Airmass & Other obs. \\
\hline Jacoby LMC 17 & 2703 & 2013 Nov. 08 & 1.33 & {$[1],[2]$} \\
MGPN LMC 35 & 2845 & 2013 Nov. 09 & 1.21 & {$[2],[3]$} \\
MGPN LMC 39 & 2103 & 2013 Dec. 22 & 1.26 & {$[2],[3]$} \\
SMP LMC 26 & 2703 & 2013 Dec. 21 & 1.29 & {$[4]$} \\
SMP LMC 31 & 2785 & 2013 Nov. 09 & 1.30 & {$[2],[4],[5]$} \\
SMP LMC 55 & 2703 & 2013 Dec. 22 & 1.29 & {$[2],[4],[5]$} \\
SMP LMC 64 & 2103 & 2013 Nov. 07 & 1.25 & {$[2],[5],[6]$} \\
SMP LMC 67 & 2733 & 2013 Dec. 23 & 1.24 & {$[2],[5],[6],[7]$} \\
SMP LMC 104A & 2703 & 2013 Nov. 10 & 1.33 & {$[7]$} \\
MGPN SMC 8 & 2703 & 2013 Nov. 08 & 1.29 & {$[3],[4],[8],[9]$} \\
SMP SMC 1 & 2103 & 2013 Nov. 08 & 1.33 & {$[1],[5],[9]$} \\
SMP SMC 12 & 2103 & 2013 Nov. 07 & 1.36 & {$[7],[9]$} \\
SMP SMC 16 & 2103 & 2013 Nov. 09 & 1.35 & {$[1],[3],[5],[6]$} \\
SMP SMC 28 & 2703 & 2013 Nov. 10 & 1.35 & {$[4],[6],[7]$} \\
\hline
\end{tabular}

References. [1] Boroson \& Liebert (1989), [2] Reid \& Parker (2006) [3] Vassiliadis et al. (1992), [4] Monk et al. (1988), [5] Meatheringham \& Dopita (1991), [6] Shaw et al. (2006), [7] Leisy \& Dennefeld (2006), [8] Shaw et al. (2010), [9] Stanghellini et al. (2003).

Table 2. Log of the standard star observations.

\begin{tabular}{lccc}
\hline \hline Name & Exp. [s] & Date & Air mass \\
\hline HILT 600 & 180 & 2013 Nov. 07 & 1.23 \\
HILT 600 & 180 & 2013 Nov. 08 & 1.23 \\
HILT 600 & 180 & 2013 Nov. 09 & 1.22 \\
EG 21 & 60 & 2013 Dec. 22 & 1.24 \\
\hline
\end{tabular}

An alternative set of parameters useful for studying the evolution of post-AGB stars is the stellar temperature and heating rate. The heating rate is a sensitive measure of the stellar mass. The pace of the temperature evolution is determined by the ratio of the stellar luminosity and remaining envelope mass.

Gesicki et al. (2014) derived heating rates from stellar temperatures and nebular ages for a sample of Galactic Bulge PNe. They needed to accelerate the evolutionary models by a factor of three to fit the white dwarf mass distribution and asteroseismological masses of central stars of PNe.

New models by Miller Bertolami (2016) confirmed the results obtained by Gesicki et al. (2014). He obtained three to ten shorter timescales of post-AGB evolution than Bloecker (1995) and Vassiliadis \& Wood (1994). His models were also brighter by $\sim 0.1-0.3$ dex for the same mass. He took advantage of an updated treatment of the constitutive microphysics and included an updated description of the mixing processes and winds.

An alternative method to derive heating rate is to measure the temperature evolution of the central star. This could be derived indirectly by comparison of the nebular line fluxes in different epochs. The change of the [O III] $5007 \AA$-to- $\mathrm{H} \beta$ flux ratio in time is sensitive to the temperature evolution of cool central stars (Hajduk et al. 2015).

In this paper, we derived heating rates for a sample of central stars in the Magellanic Clouds and compared them with the existing evolutionary models. An advantage of Magellanic Cloud objects over Galactic PNe is the precisely determined distance. Moreover, Magellanic Cloud PNe are often compact and spatially unresolved by ground based telescopes. In such cases, the ion stratification in the nebula would not affect the measured flux ratios.

\section{Observations}

We observed the fourteen PNe with the South African Large Telescope (SALT) equipped with the Robert Stobie Spectrograph (Kobulnicky et al. 2003; Burgh et al. 2003). The SALT telescope has a fixed azimuthal angle. The Magellanic Clouds were accessible with the SALT near the meridian (hour angle from -2 to 2 ), so that the observing track length was sufficiently long. Under these conditions, atmospheric refraction should not play a significant role.

Three chips recorded each spectrum in different wavelength ranges: 4345-5347 $\mathrm{A}, 5403-6393 \AA$, and 6448-7397 $\mathrm{\AA}$. We used longslit with the projected width of two arcsec. The resulting resolution was about 800 in the center of the spectral range. The slit was oriented north-south. The range of air masses for the targets and standards was 1.21-1.36 (Tables 1 and 2). The log of observations is given in Table 1.

The spectra were extracted and wavelength calibrated. We calibrated the flux scale using a standard observed during the same night. On three nights: November 10, December 21, and December 22, flux standards were not observed. For these three nights we used the sensitivity curve derived for the closest night.

We measured the emission line fluxes with the Gaussian fit. We computed the errors of individual lines according to the formulae provided by Lenz \& Ayres (1992). We added the derived error (root mean-square) of the sensitivity curve. We computed an error using at least two closest sensitivity curves if the standard was not observed during the given night. The fluxes were dereddened using the extinction law given by Fitzpatrick (1999) and the total to selective extinction ratio $R_{V}=3.1$ (Table A.1). We derived the extinction from the observed $\mathrm{H} \alpha$-to- $\mathrm{H} \beta$ line flux ratio.

\section{New [WC] central stars}

Hydrogen- and helium-burning post-AGB stars have different evolutionary tracks. Helium-burning tracks show slower evolution by approximately a factor of three in comparison to $\mathrm{H}$ burning tracks for the same remnant mass.

In order to compare the evolution of the central stars of PNe with the evolutionary tracks, one needs to know which shell is active. This can be inferred from the photospheric abundances 
of a star. A hydrogen-poor atmosphere indicates that the star is a helium burner.

Most of the H-deficient central stars show [WC] spectra (Weidmann \& Gamen 2011). They are relatively easy do identify, even if the stellar continuum is unobservable, since they show wide and prominent stellar wind lines imposed on the nebular spectrum (Acker \& Neiner 2003). A hydrogen-rich atmosphere does not unambiguously indicate which shell is the main source of the energy in the star. However, we assumed the stars that do not show emission lines to be $\mathrm{H}$ burners.

One of the stars in the sample (MGPN SMC 8) is a known [WC] central star (Pena et al. 1997). We detected three more [WC] stars in our sample: SMPLMC 31, SMPLMC 55, and SMP SMC 1. They show emission lines of C III, C IV, and He II. The He II $4686 \AA$ line in SMP LMC 31 has a full width at half maximum (FWHM) of $13 \AA$, while SMPLMC 55 and SMP SMC 1 have FWHMs of C IV $4650 \AA$ lines of $12 \AA$ and $8 \AA$, respectively. Neither of the new [WC] stars show the C IV $5806 \AA$ line. Thus, we classify them as [WC 11] type (Acker \& Neiner 2003). However, faint C II 7231 and $7236 \AA$ lines would suggest an earlier type.

\section{Heating rates}

We combined new and archival spectra of PNe to study the temperature evolution of their central stars. We used the [O III] $5007 \AA$-to- $\mathrm{H} \beta$ line ratio. This ratio is sensitive to the changes of stellar temperature for cool central stars due to a much higher ionization potential of $\mathrm{O}^{+}$than hydrogen. Both lines are relatively strong and located close to each other, so wavelengthdependent calibration errors are reduced.

We fit a linear function to the [O III] $5007 \AA$ A-to-H $\beta$ line flux ratio observed in different epochs for each PN (Fig. 1). We discarded one point by Reid \& Parker (2006) for SMP LMC 64 from the fitting, which exceeded all other points by a factor of two. The fit slope was larger than one, at least by $3 \sigma$ in five $\mathrm{PNe}$, indicating an increasing stellar temperature.

Among the PNe showing the flux evolution, one (MGPNSMC 8) was observed five times, three PNe were observed four times, and one PN has three observations. The observations show a linear increase of the [O III] $5007 \AA$-to-H $\beta$ flux ratio in time. An exception is SMPLMC 55. The third observation by Reid \& Parker (2006) is significantly higher than the last one. However, the [O III] $5007 \AA$ A-to-H $\beta$ flux ratio determined by Reid \& Parker (2006) appears to be systematically higher than fluxes measured by other authors.

We performed spherically symmetric photoionization models of these five PNe with the Cloudy v. 17.01 code. All the models were constrained by the observed absolute $\mathrm{H} \beta$ flux. We fit the nebular line flux ratios observed in 2013 (Table A.1).

The nebular sizes and stellar luminosities are available for three objects observed with the Hubble Space Telescope by Villaver et al. (2003) and Villaver et al. (2004). However, for the PN MGPN SMC 8 temperature and luminosity, determination is most likely affected by the stellar contribution to the He II $4686 \AA$ line flux (Shaw et al. 2010). For this and two other PNe, stellar luminosity was a free parameter. The adopted luminosities, or those derived from our photoionization models $L_{*}$, are given in Table 3.

We varied stellar temperature to obtain the best fit. We used atmosphere models by Castelli et al. (2003). The grid covers the temperature range of $3.5-50 \mathrm{kK}$.

We used nebular abundances by Leisy \& Dennefeld (2006) as an input parameter, but varied it to better fit the observed emission line fluxes. For some cases (e.g., MGPNLMC 39, SMP LMC 31, and SMP SMC 1) we were not able to achieve the observed [O III] $5007 \AA$-to-H $\beta$ line flux ratio with the O/H abundances derived by Leisy \& Dennefeld (2006). At low metallicities, the [O III] $5007 \AA$-to-H $\beta$ line flux ratio depends on the nebular $\mathrm{O} / \mathrm{H}$ abundance and stellar temperature (Stasińska 2007). For the average SMC oxygen abundance, the [O III] $5007 \AA$ A-to$\mathrm{H} \beta$ flux ratio reaches a maximum of five at a temperature of about $100 \mathrm{kK}$ (Stanghellini et al. 2003). For the LMC, the models reach maximum of ten at similar stellar temperatures.

Table 3 shows the input parameters and results of the photoionization modeling of the five objects. The modeled line fluxes are compared to the fluxes measured in 2013 in Table A.4.

We varied stellar temperature to fit the [O III] $5007 \AA$-to-H $\beta$ line flux ratio observed in other epochs. After we obtained the temperatures in different years, we used them to compute heating rates $\dot{T}_{*}$. The uncertainty of the measured [O III] $5007 \AA$ A-to$\mathrm{H} \beta$ line flux ratio propagates to the measured temperatures in different epochs and subsequently to the heating rates presented in Table 3.

We computed heating rates from the evolutionary tracks as a function of stellar temperature. We used models by Miller Bertolami (2016) for the star that does not show emission lines, and Vassiliadis \& Wood (1994) for four [WC] central stars. Miller Bertolami (2016) computed only three He-burning models spanning a very low range of masses.

Heating rates derived from the evolutionary tracks were interpolated to fit observed heating rates. We determined model-dependent luminosities $L_{\mathrm{MOD}}$ and corresponding modeldependent masses $M_{\mathrm{MOD}}$. The errors of $L_{\mathrm{MOD}}$ and $M_{\mathrm{MOD}}$ shown in Table 3 result from the uncertainties in $\dot{T}_{*}$.

We did not include systematic errors in measured heating rates in Table 3. These depend mostly on the determination of the $\mathrm{O} / \mathrm{H}$ abundance. For an adopted uncertainty of the $\log (\mathrm{O} / \mathrm{H})$ of 0.3 in dex, the model-dependent stellar mass $M_{\mathrm{MOD}}$ is accurate to about $0.04 M_{\odot}$, and $\log$ of luminosity to 0.15 dex. Moreover, the model-dependent masses for [WC] stars derived using tracks by Vassiliadis \& Wood (1994) may be overestimated by $0.038 M_{\odot}$ (Gesicki et al. 2014).

Heating rates derived from the evolutionary tracks do not vary strongly with stellar temperature. The uncertainty of stellar temperature determination affects the derived model-dependent parameters to a much lower extent than the uncertainty in the determination of $\mathrm{O} / \mathrm{H}$ abundance.

\section{Discussion}

We attempted to select PNe with relatively cool central stars to study their evolution. Our sample fulfils the criterion of [O III] $5007 \AA$-to-H $\beta$ ratio $\lesssim 3$ for all but one object. However, this criterion appeared to be insufficient in some cases. The [O III] $5007 \AA$ line may be weak in hot PNe, due to photoionization of $\mathrm{O}^{++}$to $\mathrm{O}^{+++}$(Marigo et al. 2001; Stasińska 1989). Also, the [O III] $5007 \AA$ line can be weak in PNe with low O abundances, despite a high temperature. This appears to be the case for three PNe in our sample: Jacoby LMC 17, MGPN LMC 35, and SMP SMC 28, which show prominent He II $4686 \AA$ lines. These PNe are not expected to show rapid evolution of the [O III] $5007 \AA$ Alux.

Young PNe can mimic symbiotic stars due to high nebular density and strong dust emission. Iłkiewicz \& Mikołajewska (2017) listed two PNe from our sample, SMPLMC 31 and SMP LMC 104A, as symbiotic star candidates. However, we did 


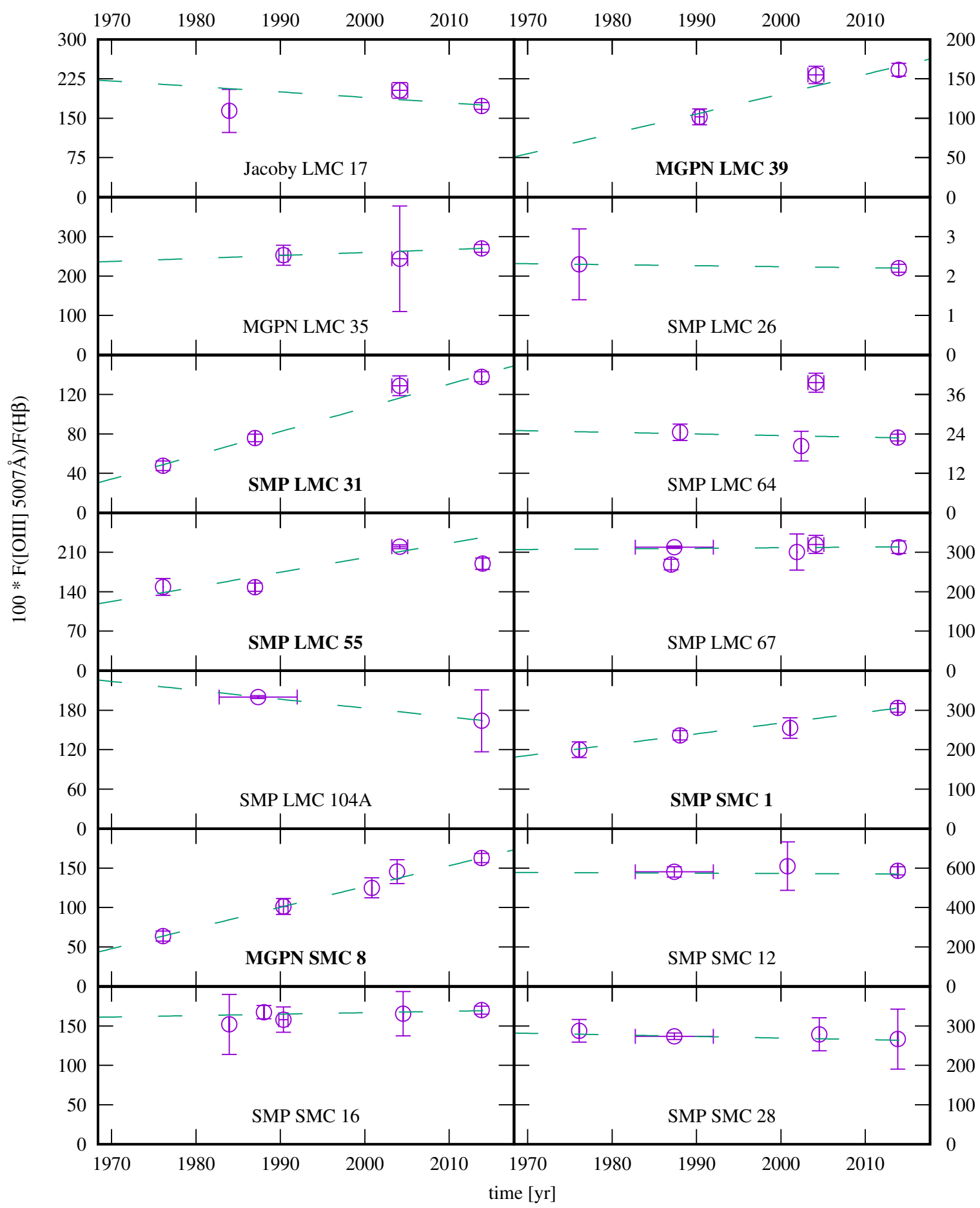

Fig. 1. Evolution of [O III $5007 \AA$ A-to-H $\beta$ line flux ratio in the Magellanic Cloud PNe. The dashed line fits the observed trend. The horizontal error bars correspond to the flux uncertainties. The vertical error bars in the time domain correspond to the observations, of which the dates were not precisely specified. Meatheringham \& Dopita (1991) did not specify dates of their observations precisely, thus the error bars in time domain are large.

not find any indication of the cool giant in their spectra. In addition, SMP LMC 31 show stellar wind lines consistent with a [WC] star.

Eleven out of fourteen PNe in our sample may be considered as young PNe. Five of them show an evolution of the [O III] $5007 \AA$ A line flux. The five central stars showing temperature evolution are most likely the most massive in our sample, and thus the most luminous. An independent luminosity determination confirms that these stars show the highest luminosities (Table 4).
The remaining six central stars may evolve too slowly to show significant evolution of the [O III] $5007 \AA$ A-to-H $\beta$ flux ratio on a timescale of decades. The collected observations are sensitive down to heating rates of about $5-10 \mathrm{~K} \mathrm{yr}^{-1}$. Lower heating rates, corresponding to the initial masses below $1.25 M_{\odot}$ (final masses below $0.532 M_{\odot}$ ) (Miller Bertolami et al. 2019), would not be detected.

Figure 2 shows the modeled evolution of nebular [O III] $5007 \AA$ A-to-H $\beta$ flux ratio with increasing stellar temperature for 
Table 3. Input parameters and results of the photoionization modeling.

\begin{tabular}{lccccc}
\hline \hline Name & MGPN LMC 39 & & \\
& & & & \\
& SMP LMC 31 & SMP LMC 55 & MGPN SMC 8 & SMP SMC 1 \\
\hline$T_{*}[\mathrm{kK}]$ & 35.7 & 34.8 & 37.3 & 37.3 & 37.2 \\
$\dot{T}_{*}\left[\mathrm{~K} \mathrm{yr}{ }^{-1}\right]$ & $19.1 \pm 4.7$ & $18.4 \pm 1.5$ & $10.4 \pm 2.1$ & $24.9 \pm 2.4$ & $17.8 \pm 3.4$ \\
$\log L_{\mathrm{MOD}} / L_{\odot}$ & $3.759 \pm 0.014$ & $3.791 \pm 0.018$ & $3.650 \pm 0.074$ & $3.867 \pm 0.029$ & $3.784 \pm 0.040$ \\
$M_{\mathrm{MOD}} / M_{\odot}$ & $0.570 \pm 0.004$ & $0.668 \pm 0.024$ & $0.613 \pm 0.014$ & $0.693 \pm 0.016$ & $0.644 \pm 0.023$ \\
$\log (\mathrm{O} / \mathrm{H})$ & -3.50 & -3.92 & -3.83 & -3.65 & -3.81 \\
$\log (\mathrm{N} / \mathrm{H})$ & & & -4.53 & & -4.62 \\
$\log L_{*} / L_{\odot}$ & $3.50[1]$ & $4.01[2]$ & $4.21[1]$ & $3.42[1]$ & $3.77[3]$ \\
$\log T_{*}(\mathrm{H} \mathrm{I})$ & $(b)$ & $28.6 \pm 2.4[2]$ & & $28.8 \pm 2.6[3]$ & $28.8 \pm 2.6[3]$ \\
$\log F(\mathrm{H} \beta)$ & $-13.07[4]$ & $-12.91[5]$ & $-12.20[4]$ & $-13.27[6]$ & $-12.85[6]$ \\
$\mathrm{d}[\operatorname{arcsec}]$ & & $0.26[5]$ & & $0.615[6]$ & $0.15[6]$ \\
\hline
\end{tabular}

Notes. Derived stellar temperatures, heating rates, model-dependent luminosities and masses for the central stars, modeled $\mathrm{O}$ and $\mathrm{N}$ abundances of the PNe, observed luminosities, temperatures, $\mathrm{H} \beta$ fluxes, and diameters. ${ }^{(a)}$ Central star without emission lines. ${ }^{(b)}$ Computed using black-body model.

References. [1] this paper, [2] Villaver et al. (2003), [3] Villaver et al. (2004), [4] Reid \& Parker (2006), [5] Stanghellini et al. (2002), [6] Stanghellini et al. (2003).

Table 4. Luminosities of the central stars of PNe obtained using different methods: model-dependent luminosities determined from heating rates, luminisities derived from Zanstra temperatures and stellar magnitudes, from the integrated spectral energy distribution, and constrained from abolute $\mathrm{H} \beta$ flux and nebular modeling.

\begin{tabular}{|c|c|c|c|c|c|c|}
\hline Ref. & $\begin{array}{c}\log L_{\mathrm{MOD}} \\
{[1]}\end{array}$ & $\begin{array}{c}\log L_{*} / L_{\odot}(\mathrm{H} \mathrm{I}) \\
{[2]}\end{array}$ & $\begin{array}{c}\log L_{\mathrm{SED}} / L_{\odot} \\
{[3]}\end{array}$ & $\begin{array}{c}\log L_{\mathrm{SED}} / L_{\odot} \\
{[4]}\end{array}$ & $\begin{array}{c}\log L_{*} / L_{\odot} \\
{[5]}\end{array}$ & $\begin{array}{c}\log L_{*} / L_{\odot} \\
{[1]}\end{array}$ \\
\hline Jacoby LMC 17 & & & 3.39 & & & \\
\hline MGPN LMC 35 & & & 3.20 & & & \\
\hline MGPN LMC 39 & 3.759 & & 3.70 & 3.47 & & 3.50 \\
\hline SMP LMC 26 & & & 3.60 & 3.32 & & \\
\hline SMP LMC 31 & 3.791 & 4.01 & 3.90 & 3.64 & & \\
\hline SMP LMC 55 & 3.650 & & & & 3.69 & 4.21 \\
\hline SMP LMC 67 & & & & 2.86 & 3.33 & \\
\hline MGPN SMC 8 & 3.867 & 4.33 & & 3.49 & & 3.42 \\
\hline SMP SMC 1 & 3.784 & 3.77 & & & & \\
\hline SMP SMC 12 & & 2.84 & & & & \\
\hline SMP SMC 16 & & & & 3.20 & & \\
\hline SMP SMC 28 & & & & & 3.28 & \\
\hline
\end{tabular}

Notes. For SMP LMC 64 and SMP LMC 104A, no data were found in the literature. The data in bold are the central stars showing temperature evolution, modeled in Table 3.

References. [1] this paper, [2] Villaver et al. (2003, 2004), [3] van Aarle et al. (2011) , [4] Kamath et al. (2014), [5] Dopita \& Meatheringham (1991).

two different $\mathrm{O} / \mathrm{H}$ abundance ratios. The [O III] $5007 \AA$ A-to-H $\beta$ flux ratio scales approximately with the $\mathrm{O}$ abundance. The flux ratio does not increase linearly throughout the whole temperature range of the central star, but it can be approximated as linear in sufficiently small temperature intervals. The heating rate is highest between 35 and $38 \mathrm{kK}$. Then it slows down. The five central stars of which the nebulae show flux evolution have stellar temperatures in this range.

The central star of MGPNLMC 39 is probably the most massive H-burning central star in our sample. Its final mass of $0.57 M_{\odot}$ is the same as the peak of the mass distribution for the central stars of Galactic PNe. The remaining H-burning central stars in our sample are less massive. This suggests that central stars in the Magellanic Clouds are less massive than the stars in the Galaxy. Hajduk et al. (2014) derived heating rates of $45 \pm 7 \mathrm{~K} \mathrm{yr}^{-1}$ for Galactic PN Hen 2-260, significantly higher than for Magellanic Cloud central stars.
We compared the model-dependent luminosities $L_{\mathrm{MOD}}$ with luminosities determined independently using different methods: using Zanstra temperatures and a black-body fit $\left(\log T_{*}(\mathrm{HI})\right)$, by fitting spectral energy distribution (SED) $\left(L_{\mathrm{SED}}\right)$ and by photoionization modeling $\left(L_{*}\right)$ (Table 4$)$. Unfortunately, the luminosities yielded from observations using different methods show a relatively large scatter.

The model-dependent luminosity agrees with the observed luminosity for SMPSMC 1 and is within the observed range of stellar luminosities for other objects. For SMP LMC 55, the model-dependent luminosity is close to the luminosity derived from photoionization modeling by Dopita \& Meatheringham (1991), but lower by 0.56 dex from the luminosity of our photoionization model. However, the absolute $\mathrm{H} \beta$ flux used by Dopita \& Meatheringham (1991) was 0.46 lower in log than that used by us from Reid \& Parker (2006). If this discrepancy were real, this would indicate an unexpected change in luminosity of this object. 


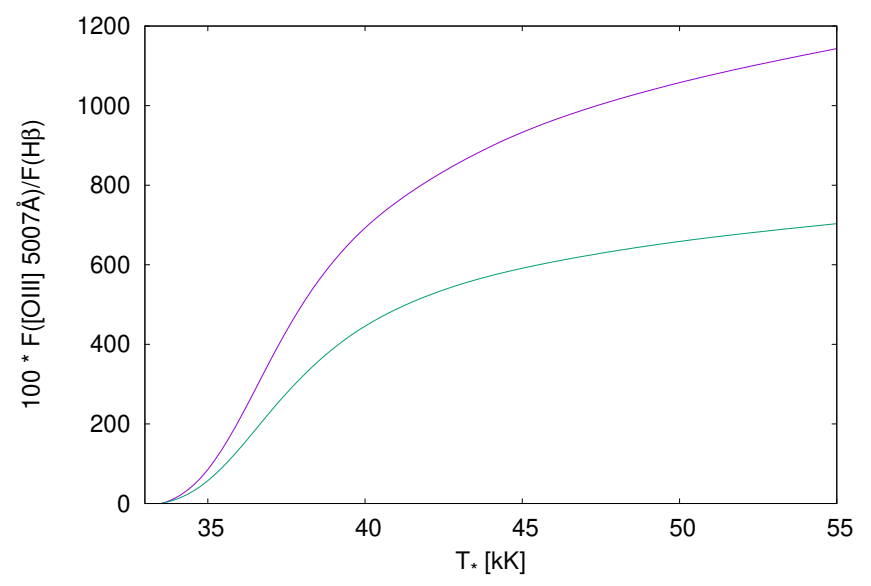

Fig. 2. Two models of the evolution of the [O III] $5007 \AA$ A-to-H $\beta$ flux ratio with the temperature of the central star in a PN. The model parameters are identical to MGPN LMC 39, and the second model the $(\mathrm{O} / \mathrm{H})$ abundance is lowered by 0.3 in dex.

We are not able to discriminate between Miller Bertolami (2016) and Vassiliadis \& Wood (1994) or Bloecker (1995). Miller Bertolami (2016) models were faster and more luminous for the same masses. However, these models all show similar evolutionary speeds for the same luminosity of the central star (but different masses). We would be able to discriminate between these models if we obtained an independent mass determination.

The [WC] central stars constitute about $15 \%$ of Galactic central stars of PNe. This ratio was suggested to be as low as 5\% in the Magellanic Clouds (Pena et al. 1997). Pena et al. (1997) reported only two [WC] central stars in the Small Magellanic Cloud (SMC) and six in the Large Magellanic Cloud (LMC). Five stars are intermediate types [WC 4] or [WC 4-5], and only one is [WC9] (Monk et al. 1988). Margon et al. (2020a) and Margon et al. (2020b) announced the discovery of two more [WC 11] stars in the LMC. They suggested that a large population of late-type [WC] stars may exist, undetected due to observational selection. Late-type [WC] stars show faint emission lines, which could be missed in observations performed with smaller telescopes. We discovered three new late-type [WC] stars.

Interestingly, the four [WC] $\mathrm{PNe}$ observed by us show rapid evolution. This implies relatively high stellar mass. Hajduk et al. (2015) observed a relatively rapid change of the [O III] $5007 \AA$ to- $\mathrm{H} \beta$ flux ratio for the Galactic PNe M 1-11 and M 1-12 containing [WC] central stars. This suggests that the evolution of Galactic [WC] stars also is faster than H-rich central stars and originate from more massive progenitors. This was independently confirmed by Weidmann \& Gamen (2011), who found that H-poor central stars are more concentrated toward the Galactic center and Galactic plane than the H-rich group. The DB white dwarfs, which are the possible progeny of [WC] central stars, show an average mass of $0.651 M_{\odot}$, higher than the $0.598 M_{\odot}$ derived for DA white dwarfs, which originate from H-rich central stars (Kleinman et al. 2013).
Acknowledgements. MH thanks the Ministry of Science and Higher Education (MSHE) of the Republic of Poland for granting funds for the Polish contribution to the International LOFAR Telescope (MSHE decision no. DIR/WK/2016/2017/05-1) and for maintenance of the LOFAR PL-612 Baldy (MSHE decision no. 59/E-383/SPUB/SP/2019.1). Polish participation in SALT is funded by the MSHE Grant No. DIR/WK/2016/07. MH acknowledges financial support from National Science Centre, Poland, grant No. 2016/23/B/ST9/01653. Some of the observations reported in this paper were obtained with the Southern African Large Telescope (SALT) under programme 2013-2-POL_OTH-001 (PI M. Hajduk).

\section{References}

Acker, A., \& Neiner, C. 2003, A\&A, 403, 659

Blöcker, T. 2001, Ap\&SS, 275, 1

Bloecker, T. 1995, A\&A, 299, 755

Boroson, T. A., \& Liebert, J. 1989, ApJ, 339, 844

Burgh, E. B., Nordsieck, K. H., Kobulnicky, H. A., et al. 2003, in Prime Focus Imaging Spectrograph for the Southern African Large Telescope: Optical Design, eds. M. Iye, \& A. F. M. Moorwood, SPIE Conf. Ser., 4841, 1463

Castelli, F., \& Kurucz, R. L. 2003, in Modelling of Stellar Atmospheres, eds. N. Piskunov, W. W. Weiss, \& D. F. Gray, IAU Symp., 210, A20

Dopita, M. A., \& Meatheringham, S. J. 1991, ApJ, 367, 115

Fitzpatrick, E. L. 1999, PASP, 111, 63

Gesicki, K., Zijlstra, A. A., Hajduk, M., \& Szyszka, C. 2014, A\&A, 566, A48

Hajduk, M., van Hoof, P. A. M., Gesicki, K., et al. 2014, A\&A, 567, A15

Hajduk, M., van Hoof, P. A. M., \& Zijlstra, A. A. 2015, A\&A, 573, A65

Herwig, F. 2005, ARA\&A, 43, 435

Iben, I. Jr., \& Renzini, A. 1983, ARA\&A, 21, 271

Iłkiewicz, K., \& Mikołajewska, J. 2017, A\&A, 606, A110

Kamath, D., Wood, P. R., \& Van Winckel, H. 2014, MNRAS, 439, 2211

Karakas, A. I. 2010, MNRAS, 403, 1413

Kleinman, S. J., Kepler, S. O., Koester, D., et al. 2013, ApJS, 204, 5

Kobulnicky, H. A., Nordsieck, K. H., Burgh, E. B., et al. 2003, in Prime Focus Imaging Spectrograph for the Southern African Large Telescope: Operational Modes, eds. M. Iye, \& A. F. M. Moorwood, SPIE Conf. Ser., 4841, 1634 Koesterke, L. 2001, Ap\&SS, 275, 41

Leisy, P., \& Dennefeld, M. 2006, A\&A, 456, 451

Lenz, D. D., \& Ayres, T. R. 1992, PASP, 104, 1104

Margon, B., Manea, C., Williams, R., et al. 2020a, ApJ, 888, 54

Margon, B., Massey, P., Neugent, K. F., \& Morrell, N. 2020b, ApJ, 898, 85

Marigo, P., Girardi, L., Groenewegen, M. A. T., \& Weiss, A. 2001, A\&A, 378, 958

Meatheringham, S. J., \& Dopita, M. A. 1991, ApJS, 75, 407

Miller Bertolami, M. M. 2016, A\&A, 588, A25

Miller Bertolami, M. M. 2019, in IAU Symposium, eds. F. Kerschbaum, M. Groenewegen, \& H. Olofsson, IAU Symp., 343, 36

Monk, D. J., Barlow, M. J., \& Clegg, R. E. S. 1988, MNRAS, 234, 583

Paczyński, B. 1970, Acta Astron., 20, 47

Pena, M., Ruiz, M. T., \& Torres-Peimbert, S. 1997, A\&A, 324, 674

Reid, W. A., \& Parker, Q. A. 2006, MNRAS, 373, 521

Schoenberner, D. 1979, A\&A, 79, 108

Shaw, R. A., Stanghellini, L., Villaver, E., \& Mutchler, M. 2006, ApJS, 167, 201

Shaw, R. A., Lee, T.-H., Stanghellini, L., et al. 2010, ApJ, 717, 562

Stanghellini, L., Shaw, R. A., Mutchler, M., et al. 2002, ApJ, 575, 178

Stanghellini, L., Shaw, R. A., Balick, B., et al. 2003, ApJ, 596, 997

Stasińska, G. 1989, A\&A, 213, 274

Stasińska, G. 2007, ArXiv e-prints [arXiv:0704 . 0348]

van Aarle, E., van Winckel, H., Lloyd Evans, T., et al. 2011, A\&A, 530, A90

Vassiliadis, E., \& Wood, P. R. 1994, ApJS, 92, 125

Vassiliadis, E., Dopita, M. A., Morgan, D. H., \& Bell, J. F. 1992, ApJS, 83, 87

Villaver, E., Stanghellini, L., \& Shaw, R. A. 2003, ApJ, 597, 298

Villaver, E., Stanghellini, L., \& Shaw, R. A. 2004, ApJ, 614, 716

Weidmann, W. A., \& Gamen, R. 2011, A\&A, 526, A6

Werner, K., \& Herwig, F. 2006, PASP, 118, 183 


\section{Appendix A: Line fluxes}

Table A.1. Measured and dereddened line fluxes in the LMC PNe with the relevant uncertainties.

\begin{tabular}{|c|c|c|c|c|c|c|c|c|c|c|c|c|c|c|c|c|}
\hline & \multirow[b]{2}{*}{$\lambda$} & \multicolumn{3}{|c|}{ Jacoby LMC 17} & \multicolumn{3}{|c|}{ MGPN LMC 35} & \multicolumn{3}{|c|}{ MGPN LMC 39} & \multicolumn{3}{|c|}{ SMP LMC 26} & \multicolumn{3}{|c|}{ SMP LMC 31} \\
\hline & & $F(\lambda)$ & $I(\lambda)$ & $\delta I$ & $F(\lambda)$ & $I(\lambda)$ & $\delta I$ & $F(\lambda)$ & $I(\lambda)$ & $\delta I$ & $F(\lambda)$ & $I(\lambda)$ & $\delta I$ & $F(\lambda)$ & $I(\lambda)$ & $\delta I$ \\
\hline$E(B-V)$ & & & 0.36 & & & 0.10 & & & 0.45 & & & 0.19 & & & 0.37 & \\
\hline $\mathrm{H} \gamma$ & 4343 & 38.6 & 45.5 & 1.7 & 43.7 & 45.8 & 3.4 & & & & & & & 43.2 & 51.1 & 1.9 \\
\hline [O III $]$ & 4363 & 20.6 & 24.1 & 1.0 & 30.1 & 31.5 & 3.3 & & & & & & & 0.9 & 1.1 & 0.2 \\
\hline He I & 4388 & & & & & & & & & & & & & 0.4 & 0.5 & 0.1 \\
\hline He I & 4471 & & & & & & & 2.6 & 3.1 & 0.4 & & & & 2.8 & 3.2 & 0.2 \\
\hline He II & 4541 & 2.0 & 2.2 & 0.3 & & & & & & & & & & 1.5 & 1.6 & 0.2 \\
\hline C IV & 4650 & & & & & & & $1.4^{(a)}$ & $1.6^{(a)}$ & $0.3^{(a)}$ & & & & $1.5^{(a)}$ & $1.6^{(a)}$ & $0.2^{(a)}$ \\
\hline He II & 4686 & 10.2 & 10.8 & 0.6 & 58.3 & 59.2 & 2.7 & & & & & & & & & \\
\hline [Ar IV]/HeI & 4712 & 1.0 & 1.1 & 0.2 & & & & & & & & & & 0.5 & 0.5 & 0.1 \\
\hline$[\mathrm{Ne}$ IV $]$ & 4726 & 1.4 & 1.4 & 0.3 & & & & & & & & & & & & \\
\hline [Ar IV] & 4741 & 0.7 & 0.7 & 0.2 & & & & & & & & & & & & \\
\hline $\mathrm{H} \beta$ & 4861 & 100.0 & 100.0 & 3.6 & 100.0 & 100.0 & 4.0 & 100.0 & 100.0 & 5.0 & 100.0 & 100.0 & 5.0 & 100.0 & 100.0 & 3.6 \\
\hline $\mathrm{He} \mathrm{I}$ & 4922 & & & & & & & & & & & & & 0.9 & 0.9 & 0.1 \\
\hline [O III] & 4931 & & & & & & & & & & & & & & & \\
\hline [O III] & 4959 & 65.1 & 63.0 & 2.3 & 88.5 & 87.7 & 3.5 & 54.7 & 52.5 & 2.7 & 0.5 & 0.5 & 0.1 & 48.7 & 47.1 & 1.7 \\
\hline [O III] & 5007 & 182.0 & 173.4 & 6.2 & 274.2 & 270.5 & 9.9 & 170.0 & 160.1 & 8.0 & 2.3 & 2.2 & 0.1 & 145.6 & 138.6 & 5.0 \\
\hline$[\mathrm{NI}]$ & 5197 & & & & & & & & & & 0.6 & 0.6 & 0.1 & 0.3 & 0.3 & 0.1 \\
\hline He II & 5412 & 6.2 & 5.2 & 0.2 & 6.4 & 6.1 & 0.8 & & & & & & & & & \\
\hline [Cl III] & 5537 & & & & & & & & & & & & & 0.1 & 0.1 & 0.1 \\
\hline C III & 5696 & & & & & & & & & & & & & $0.8^{(a)}$ & $0.6^{(a)}$ & $0.1^{(a)}$ \\
\hline [N II] & 5755 & 4.1 & 3.2 & 0.2 & & & & 2.2 & 1.6 & 0.2 & 2.7 & 2.4 & 0.1 & 3.6 & 2.8 & 0.2 \\
\hline He I & 5876 & 5.3 & 4.0 & 0.2 & 10.9 & 10.1 & 1.0 & 12.4 & 8.7 & 0.5 & 1.8 & 1.5 & 0.1 & 13.7 & 10.3 & 0.4 \\
\hline [O I] & 6302 & 13.2 & 9.1 & 0.3 & 5.2 & 4.7 & 0.5 & 2.6 & 1.6 & 0.2 & 2.3 & 1.9 & 0.1 & 3.3 & 2.2 & 0.1 \\
\hline [S III] & 6312 & 2.7 & 1.9 & 0.1 & & & & 2.1 & 1.3 & 0.1 & & & & 1.0 & 0.7 & 0.1 \\
\hline [O I] & 6347 & & & & & & & & & & & & & 0.3 & 0.2 & 0.1 \\
\hline [O I] & 6365 & 4.7 & 3.2 & 0.1 & 3.9 & 3.5 & 0.7 & 0.7 & 0.4 & 0.1 & 0.5 & 0.5 & 0.1 & 1.2 & 0.8 & 0.1 \\
\hline [N II] & 6548 & 15.2 & 10.0 & 0.4 & 14.1 & 12.5 & 0.7 & 15.7 & 9.3 & 0.5 & 41.6 & 33.6 & 1.7 & 23.6 & 15.5 & 0.6 \\
\hline $\mathrm{H} \alpha$ & 6563 & 433.5 & 286.0 & 10.1 & 321.9 & 286.0 & 10.2 & 482.6 & 286.0 & 14.2 & 355.0 & 286.0 & 14.2 & 438.5 & 286.0 & 10.2 \\
\hline$[\mathrm{N}$ II $]$ & 6584 & 34.5 & 22.7 & 0.8 & 28.3 & 25.1 & 1.1 & 42.9 & 25.3 & 1.3 & 119.0 & 95.7 & 4.7 & 71.7 & 46.6 & 1.7 \\
\hline He I & 6678 & 1.7 & 1.1 & 0.1 & 2.9 & 2.6 & 0.5 & 3.9 & 2.3 & 0.2 & 0.6 & 0.5 & 0.9 & 4.3 & 2.8 & 0.2 \\
\hline [S II] & 6716 & 2.0 & 1.3 & 0.1 & 4.8 & 4.2 & 0.6 & 3.9 & 0.6 & 0.1 & 0.4 & 0.3 & 0.1 & 0.5 & 0.3 & 0.1 \\
\hline [S II] & 6730 & 2.4 & 1.5 & 0.1 & 4.5 & 4.0 & 0.5 & & & & 1.7 & 1.4 & 0.1 & 1.1 & 0.7 & 0.1 \\
\hline He I & 7002 & & & & & & & & & & & & & & & \\
\hline [Ar V] & 7007 & 3.1 & 1.9 & 0.1 & 4.0 & 3.5 & 0.5 & 11.4 & 6.1 & 0.4 & 0.4 & 0.3 & 0.1 & & & \\
\hline $\mathrm{He} \mathrm{I}$ & 7065 & 3.3 & 2.0 & 0.1 & 7.3 & 6.4 & 0.6 & & & & 0.8 & 0.6 & 0.1 & & & \\
\hline [Ar III] & 7100 & & & & & & & 11.2 & 5.9 & 0.3 & & & & 0.5 & 0.3 & 0.1 \\
\hline [Ar III] & 7136 & 7.2 & 4.3 & 0.2 & 7.4 & 6.4 & 0.7 & & & & 1.0 & 0.8 & 0.1 & 9.2 & 5.4 & 0.2 \\
\hline [Ar IV] & 7172 & 1.5 & 0.9 & 0.1 & & & & 2.4 & 1.3 & 0.1 & & & & & & \\
\hline [Ar IV] & 7237 & & & & & & & & & & & & & 2.0 & 1.2 & 0.1 \\
\hline $\mathrm{He} \mathrm{I}$ & 7281 & & & & & & & 1.0 & 0.5 & 0.1 & & & & 1.1 & 0.6 & 0.1 \\
\hline [O II] & 7320 & 8.5 & 5.0 & 0.2 & & & & 65.9 & 33.5 & 1.7 & 23.4 & 17.7 & 0.9 & 46.2 & 26.6 & 1.0 \\
\hline [O II] & 7330 & 6.9 & 4.0 & 0.2 & & & & 49.0 & 24.9 & 1.3 & 17.6 & 13.3 & 0.7 & 36.8 & 21.1 & 0.8 \\
\hline
\end{tabular}

Notes. The value of the $E(B-V)$ color excess applied for dereddening the fluxes is given for each PN. ${ }^{(a)}$ Stellar line. 
Table A.2. Measured and dereddened line fluxes in the MC PNe with the relevant uncertainties.

\begin{tabular}{|c|c|c|c|c|c|c|c|c|c|c|c|c|c|c|c|c|}
\hline & \multirow[b]{2}{*}{$\lambda$} & \multicolumn{3}{|c|}{ SMP LMC 55} & \multicolumn{3}{|c|}{ SMP LMC 64} & \multicolumn{3}{|c|}{ SMP LMC 67} & \multicolumn{3}{|c|}{ SMP LMC 104A } & \multicolumn{3}{|c|}{ MGPN SMC 8} \\
\hline & & $F(\lambda)$ & $I(\lambda)$ & $\delta I$ & $F(\lambda)$ & $I(\lambda)$ & $\delta I$ & $F(\lambda)$ & $I(\lambda)$ & $\delta I$ & $F(\lambda)$ & $I(\lambda)$ & $\delta I$ & $F(\lambda)$ & $I(\lambda)$ & $\delta I$ \\
\hline$E(B-V)$ & & & 0.25 & & & 0.52 & & & 0.22 & & & 0.23 & & & 0.26 & \\
\hline $\mathrm{H} \gamma$ & 4343 & & & & 56.5 & 71.8 & 1.9 & & & & 51.0 & 56.7 & 16.1 & 35.1 & 39.5 & 1.5 \\
\hline [O III] & 4363 & 1.3 & 1.5 & 0.2 & 3.2 & 4.1 & 0.2 & 1.4 & 1.6 & 0.2 & 21.1 & 23.2 & 6.6 & 2.3 & 2.6 & 0.3 \\
\hline He I & 4388 & 0.5 & 0.6 & 0.1 & 1.0 & 1.3 & 0.2 & 0.6 & 0.6 & 0.1 & 0.2 & 0.2 & 0.1 & & & \\
\hline $\mathrm{He} \mathrm{I}$ & 4471 & 3.4 & 3.7 & 0.2 & 4.4 & 5.3 & 0.2 & 4.3 & 4.5 & 0.3 & 1.5 & 1.5 & 0.5 & 4.4 & 4.8 & 0.3 \\
\hline He II & 4541 & 0.4 & 0.4 & 0.1 & 1.4 & 1.6 & 0.1 & & & & 2.2 & 2.3 & 0.7 & 1.9 & 2.0 & 0.2 \\
\hline CIV & 4650 & $1.1^{(a)}$ & $1.1^{(a)}$ & $0.1^{(a)}$ & & & & & & & & & & $119.5^{(a)}$ & $125.6^{(a)}$ & $4.5^{(a)}$ \\
\hline He II & 4686 & $1.1^{(a)}$ & $1.1^{(a)}$ & $0.1^{(a)}$ & & & & & & & 62.2 & 65.0 & 18.4 & $24.9^{(a)}$ & $26.0^{(a)}$ & $1.0^{(a)}$ \\
\hline$[\mathrm{Ar}$ IV]/HeI & 4712 & 0.5 & 0.6 & 0.1 & 0.5 & 0.6 & 0.1 & 0.6 & 0.6 & 0.1 & 1.5 & 1.5 & 0.5 & & & \\
\hline [Ne IV] & 4726 & & & & & & & & & & 1.5 & 1.5 & 0.5 & & & \\
\hline [Ar IV] & 4741 & & & & & & & & & & 1.1 & 1.1 & 0.4 & & & \\
\hline $\mathrm{H} \beta$ & 4861 & 100.0 & 100.0 & 5.0 & 100.0 & 100.0 & 2.6 & 100.0 & 100.0 & 5.0 & 100.0 & 100.0 & 28.3 & 100.0 & 100.0 & 3.6 \\
\hline He I & 4922 & 0.9 & 0.8 & 0.1 & 2.4 & 2.3 & 0.1 & 1.3 & 1.3 & 0.1 & 0.6 & 0.6 & 0.2 & 1.0 & 1.0 & 0.2 \\
\hline [O III] & 4931 & & & & & & & & & & 0.5 & 0.5 & 0.2 & & & \\
\hline [O III] & 4959 & 64.4 & 62.9 & 3.2 & 6.3 & 6.1 & 0.2 & 105.1 & 103.4 & 5.2 & 56.8 & 54.4 & 15.4 & 53.7 & 52.5 & 1.9 \\
\hline [O III] & 5007 & 193.8 & 187.3 & 9.3 & 22.8 & 21.3 & 0.6 & 320.4 & 303.7 & 15.1 & 172.1 & 163.8 & 46.4 & 168.2 & 162.5 & 5.8 \\
\hline$[\mathrm{NI}]$ & 5197 & 0.5 & 0.4 & 0.1 & 1.3 & 1.1 & 0.1 & 1.4 & 1.4 & 0.1 & & & & & & \\
\hline He II & 5412 & & & & & & & & & & & & & & & \\
\hline [Cl III] & 5537 & 0.2 & 0.2 & 0.1 & & & & 0.3 & 0.2 & 0.1 & & & & & & \\
\hline C III & 5696 & $0.4^{(a)}$ & $0.3^{(a)}$ & $0.1^{(a)}$ & & & & & & & & & & $51.1^{(a)}$ & $43.0^{(a)}$ & $1.5^{(a)}$ \\
\hline [N II] & 5755 & 2.3 & 1.9 & 0.1 & & & & 4.1 & 3.5 & 0.2 & 0.7 & 0.6 & 0.2 & & & \\
\hline He I & 5876 & 13.3 & 10.9 & 0.6 & & & & 16.0 & 12.9 & 0.7 & 5.0 & 4.2 & 1.2 & & & \\
\hline [OI] & 6302 & 2.8 & 2.2 & 0.2 & 6.4 & 3.8 & 0.1 & 2.1 & 1.7 & 0.1 & 2.9 & 2.3 & 0.7 & 6.8 & 5.2 & 0.2 \\
\hline [S III] & 6312 & 1.2 & 0.9 & 0.1 & 5.1 & 3.0 & 0.1 & 0.7 & 0.6 & 0.1 & 1.6 & 1.2 & 0.4 & 1.1 & 0.9 & 0.1 \\
\hline [O I] & 6347 & & & & & & & & & & & & & & & \\
\hline [O I] & 6365 & 0.9 & 0.7 & 0.1 & 2.1 & 1.2 & 0.1 & 0.7 & 0.6 & 0.1 & 0.9 & 0.7 & 0.3 & 2.3 & 1.7 & 0.1 \\
\hline$[\mathrm{N}$ II $]$ & 6548 & 34.0 & 25.4 & 1.3 & 37.4 & 20.5 & 0.6 & 102.3 & 80.4 & 4.0 & 5.3 & 4.1 & 1.2 & 37 & 27.6 & 1.0 \\
\hline $\mathrm{H} \alpha$ & 6563 & 384.3 & 286.0 & 14.2 & 522.8 & 286.0 & 7.2 & 364.8 & 286.0 & 14.2 & 372.3 & 286.0 & 80.9 & 385.8 & 286.0 & 10.2 \\
\hline [N II] & 6584 & 102.3 & 75.9 & 3.8 & 50.3 & 27.3 & 0.7 & 309.6 & 240.3 & 11.9 & 3.0 & 2.3 & 0.7 & 116.3 & 86.0 & 3.1 \\
\hline $\mathrm{He} \mathrm{I}$ & 6678 & 4.0 & 2.9 & 0.2 & 2.7 & 1.4 & 0.1 & 4.7 & 3.7 & 0.2 & 0.9 & 0.7 & 0. & 0.9 & 0.7 & 0.1 \\
\hline [S II] & 6716 & 1.7 & 1.2 & 0.1 & 0.6 & 0.3 & 0.1 & 3.4 & 2.6 & 0.2 & 0.3 & 0.2 & 0.1 & 10.7 & 7.8 & 0.3 \\
\hline$[\mathrm{S}$ II $]$ & 6730 & 3.4 & 2.4 & 0.2 & 0.9 & 0.5 & 0.1 & 5.0 & 3.8 & 0.2 & 0.5 & 0.3 & 0.1 & 14.4 & 10.5 & 0.4 \\
\hline He I & 7002 & 0.2 & 0.1 & 0.3 & 0.5 & 0.2 & 0.1 & 0.1 & 0.1 & 0.1 & & & & & & \\
\hline$[\mathrm{Arv}]$ & 7007 & & & & & & & & & & 2.3 & 1.7 & 0.5 & & & \\
\hline He I & 7065 & 9.9 & 6.9 & 2.3 & 4.6 & 2.2 & 0.1 & 6.2 & 4.6 & 0.3 & 3.8 & 2.8 & 0.8 & 6.7 & 4.7 & 0.2 \\
\hline $\begin{array}{l}\text { [Ar III] } \\
{[\mathrm{Ar} \mathrm{III]}}\end{array}$ & $\begin{array}{l}7100 \\
7136\end{array}$ & 28.7 & 6.3 & & 5.7 & 2.7 & & & & & & & & & & 02 \\
\hline [Ar IV] & 7172 & 21.8 & 0.7 & $\begin{array}{l}2.1 \\
0.5\end{array}$ & 0.4 & $\begin{array}{l}2.1 \\
0.2\end{array}$ & $\begin{array}{l}0.1 \\
0.1\end{array}$ & 9.2 & 6.7 & 0.4 & $\begin{array}{l}3.1 \\
0.6\end{array}$ & $\begin{array}{l}2.2 \\
0.5\end{array}$ & $\begin{array}{l}0.7 \\
0.2\end{array}$ & 6.6 & 4.6 & 0.2 \\
\hline [Ar IV] & 7237 & & & & & & & & & & & & & & & \\
\hline He I & 7281 & 1.0 & 0.7 & 0.5 & 0.7 & 0.3 & 0.1 & 0.9 & 0.7 & 0.1 & 0.3 & 0.2 & 0.1 & & & \\
\hline [O II] & 7320 & 28.7 & 19.6 & 6.0 & 24.5 & 11.2 & 0.3 & 7.1 & 5.2 & 0.3 & 3.8 & 2.8 & 0.8 & 16.0 & 10.8 & 0.4 \\
\hline [O II] & 7330 & 21.8 & 14.9 & 4.8 & 19.2 & 8.8 & 0.3 & 6.0 & 4.4 & 0.3 & 3.1 & 2.2 & 0.7 & 12.0 & 8.2 & 0.3 \\
\hline
\end{tabular}

Notes. The value of the $E(B-V)$ color excess applied for dereddening the fluxes is given for each PN. ${ }^{(a)}$ Stellar line 
M. Hajduk: Rapid evolution of [WC] stars in the Magellanic Clouds

Table A.3. Measured and dereddened line fluxes in the SMC PNe with the relevant uncertainties.

\begin{tabular}{|c|c|c|c|c|c|c|c|c|c|c|c|c|c|}
\hline & \multirow[b]{2}{*}{$\lambda$} & \multicolumn{3}{|c|}{ SMP SMC 1} & \multicolumn{3}{|c|}{ SMP SMC 12} & \multicolumn{3}{|c|}{ SMP SMC 16} & \multicolumn{3}{|c|}{ SMP SMC 28} \\
\hline & & $F(\lambda)$ & $I(\lambda)$ & $\delta I$ & $F(\lambda)$ & $I(\lambda)$ & $\delta I$ & $F(\lambda)$ & $I(\lambda)$ & $\delta I$ & $F(\lambda)$ & $I(\lambda)$ & $\delta I$ \\
\hline$E(B-V)$ & & & 0.03 & & & 0.00 & & & 0.13 & & & 0.06 & \\
\hline $\mathrm{H} \gamma$ & 4343 & & & & 54.0 & 54.0 & 2.3 & 37.9 & 40.6 & 1.3 & 41.9 & 43.1 & 12.2 \\
\hline [O III] & 4363 & 2.4 & 2.4 & 0.2 & 8.4 & 8.4 & 0.9 & & & & 12.3 & 12.6 & 3.6 \\
\hline He I & 4388 & 0.6 & 0.6 & 0.1 & & & & & & & & & \\
\hline He I & 4471 & 4.6 & 4.7 & 0.2 & 3.0 & 3.0 & 0.5 & 3.3 & 3.5 & 0.2 & 6.1 & 6.3 & 1.8 \\
\hline He II & 4541 & & & & & & & & & & 1.9 & 1.9 & 0.6 \\
\hline C IV & 4650 & $2.0^{(a)}$ & $2.0^{(a)}$ & $0.2^{(a)}$ & & & & & & & 1.3 & 1.3 & 0.4 \\
\hline He II & 4686 & 0.4 & 0.4 & 0.1 & & & & & & & 58.4 & 59.0 & 16.7 \\
\hline [Ar IV]/HeI & 4712 & 0.7 & 0.8 & 0.1 & 1.6 & 1.6 & 0.4 & 0.8 & 0.8 & 0.2 & 5.3 & 5.4 & 1.6 \\
\hline$[\mathrm{Ne}$ IV] & 4726 & & & & & & & & & & 1.5 & 1.5 & 0.5 \\
\hline [Ar IV] & 4741 & & & & & & & & & & 4.9 & 4.9 & 1.4 \\
\hline $\mathrm{H} \beta$ & 4861 & 100.0 & 100.0 & 3.6 & 100.0 & 100.0 & 3.7 & 100.0 & 101.1 & 2.9 & 100.0 & 100.0 & 28.3 \\
\hline He I & 4922 & 0.8 & 0.8 & 0.1 & 0.6 & 0.6 & 0.3 & 1.0 & 1.0 & 0.1 & 1.4 & 1.4 & 0.4 \\
\hline [O III] & 4931 & & & & & & & & & & & & \\
\hline [O III] & 4959 & 101.5 & 101.3 & 3.6 & 197.2 & 197.2 & 7.1 & 57.7 & 57.7 & 1.7 & 89.8 & 89.3 & 25.3 \\
\hline [O III] & 5007 & 304.5 & 303.4 & 10.8 & 591.5 & 591.5 & 21.1 & 171.7 & 170.7 & 4.9 & 267.4 & 265.3 & 75.1 \\
\hline$[\mathrm{N} \mathrm{I}]$ & 5197 & & & & & & & & & & 2.2 & 2.1 & 0.7 \\
\hline He II & 5412 & & & & & & & & & & 3.9 & 3.8 & 1.1 \\
\hline [Cl III] & 5537 & & & & & & & 0.2 & 0.1 & 0.1 & & & \\
\hline C III & 5696 & $0.3^{(a)}$ & $0.3^{(a)}$ & $0.1^{(a)}$ & & & & & & & & & \\
\hline$[\mathrm{N}$ II $]$ & 5755 & 1.1 & 1.1 & 0.1 & & & & 0.8 & 0.7 & 0.1 & 8.5 & 8.1 & 2.4 \\
\hline He I & 5876 & 14.1 & 13.8 & 0.5 & 14.6 & 14.6 & 0.6 & 12.7 & 11.7 & 0.4 & & & \\
\hline [O I] & 6302 & 1.8 & 1.8 & 0.1 & & & & 1.6 & 1.4 & 0.1 & 3.1 & 2.9 & 0.9 \\
\hline [S III] & 6312 & 0.7 & 0.7 & 0.1 & & & & 0.5 & 0.5 & 0.1 & 2.9 & 2.7 & 0.8 \\
\hline$[\mathrm{OI}]$ & 6347 & 0.1 & 0.1 & 0.1 & & & & & & & & & \\
\hline [O I] & 6365 & 0.6 & 0.6 & 0.1 & & & & 0.6 & 0.6 & 0.1 & 0.9 & 0.9 & 0.3 \\
\hline [N II] & 6548 & 8.4 & 8.2 & 0.3 & & & & 14.3 & 12.5 & 0.4 & 62.1 & 58.0 & 16.4 \\
\hline $\mathrm{H} \alpha$ & 6563 & 295.5 & 286.0 & 10.2 & 270.4 & 270.4 & 9.6 & 327.2 & 286.0 & 8.1 & 306.5 & 286.0 & 80.9 \\
\hline$[\mathrm{N}$ II $]$ & 6584 & 22.9 & 22.1 & 0.8 & 1.4 & 1.4 & 0.2 & 41.3 & 36.0 & 1.1 & 174.2 & 162.4 & 46.0 \\
\hline He I & 6678 & 3.6 & 3.5 & 0.2 & 3.7 & 3.7 & 0.2 & 3.6 & 3.1 & 0.1 & 4.9 & 4.5 & 1.3 \\
\hline [S II $]$ & 6716 & 0.3 & 0.3 & 0.1 & & & & 1.3 & 1.1 & 0.1 & 3.9 & 3.6 & 1.1 \\
\hline [S II] & 6730 & 0.6 & 0.6 & 0.1 & & & & 2.5 & 2.1 & 0.1 & 6.8 & 6.3 & 1.8 \\
\hline $\mathrm{He} I$ & 7002 & 0.2 & 0.2 & 0.1 & & & & & & & & & \\
\hline [Ar V] & 7007 & & & & & & & & & & 2.2 & 2.0 & 0.6 \\
\hline $\mathrm{He} \mathrm{I}$ & 7065 & 10.3 & 9.9 & 0.4 & 3.9 & 3.9 & 0.3 & 8.2 & 7.0 & 0.3 & 12.5 & 11.5 & 3.3 \\
\hline [Ar III] & 7100 & 0.3 & 0.3 & 0.1 & & & & & & & & & \\
\hline [Ar III] & 7136 & 5.2 & 5.0 & 0.2 & 1.9 & 1.9 & 0.2 & 3.4 & 2.8 & 0.1 & 9.1 & 8.4 & 2.4 \\
\hline [Ar IV] & 7172 & & & & & & & & & & 1.4 & 1.3 & 0.4 \\
\hline [Ar IV] & 7237 & 1.7 & 1.6 & 0.1 & & & & 0.8 & 0.7 & 0.1 & & & \\
\hline He I & 7281 & 1.0 & 1.0 & 0.1 & & & & 1.0 & 0.9 & 0.1 & 1.3 & 1.2 & 0.4 \\
\hline [O II] & 7320 & 16.4 & 15.7 & 0.6 & & & & 13.2 & 11.1 & 0.4 & 4.2 & 3.8 & 1.1 \\
\hline [O II] & 7330 & 13.3 & 12.7 & 0.5 & & & & 10.2 & 8.5 & 0.3 & 3.4 & 3.1 & 0.9 \\
\hline
\end{tabular}

Notes. The value of the $E(B-V)$ color excess applied for dereddening the fluxes is given for each PN. ${ }^{(a)}$ Stellar line. 
Table A.4. Modeled and observed line-flux ratios in the five MC PNe showing the evolution of the [O III] $5007 \AA$ Aine flux.

\begin{tabular}{|c|c|c|c|c|c|c|c|c|c|c|c|}
\hline \multirow[b]{2}{*}{ ID } & \multirow[b]{2}{*}{$\lambda$} & \multicolumn{2}{|c|}{ MGPN LMC 39} & \multicolumn{2}{|c|}{ SMP LMC 31} & \multicolumn{2}{|c|}{ SMP LMC 55} & \multicolumn{2}{|c|}{ MGPN SMC 8} & \multicolumn{2}{|c|}{ SMP SMC 1} \\
\hline & & Model & Observed & Model & Observed & Model & Observed & Model & Observed & Model & Observed \\
\hline [O III] & 4363 & & & 0.8 & 1.1 & 1.4 & 1.5 & 1.3 & 2.6 & 2.5 & 2.4 \\
\hline $\mathrm{He} \mathrm{I}$ & 4471 & 2.1 & 3.1 & 1.7 & 3.2 & 2.9 & 3.7 & 2.7 & 4.8 & 3.4 & 4.7 \\
\hline He I & 4713 & & 0.6 & 0.2 & 0.5 & 0.4 & 0.6 & & & 0.5 & 0.8 \\
\hline $\mathrm{H} \beta$ & 4861 & 100.0 & 100.0 & 100.0 & 100.0 & 100.0 & 100 & 100.0 & 100.0 & 100.0 & 100.0 \\
\hline $\mathrm{He} \mathrm{I}$ & 4922 & & & 0.5 & 0.9 & 0.8 & 0.8 & 0.7 & 1.0 & 0.9 & 0.8 \\
\hline [O III] & 4959 & 47.9 & 52.5 & 42.0 & 47.1 & 58.7 & 62.9 & 63.5 & 52.5 & 99.2 & 101.5 \\
\hline [O III] & 5006 & 143.0 & 160.1 & 125.4 & 138.6 & 175.1 & 187.3 & 189.6 & 162.5 & 296.0 & 303.4 \\
\hline$[\mathrm{NI}]$ & 5197 & & & 0.0 & 0.3 & 1.8 & 0.4 & & & & \\
\hline$[\mathrm{N}$ II $]$ & 5755 & 1.0 & 1.6 & 0.6 & 2.8 & 8.4 & 1.9 & & & 1.9 & 1.1 \\
\hline He I & 5876 & 6.0 & 8.7 & 4.3 & 10.3 & & & & & & \\
\hline [O I] & 6300 & 3.8 & 1.6 & 1.6 & 2.2 & 3.1 & 2.2 & 4.2 & 5.2 & 2.9 & 1.8 \\
\hline [S III] & 6312 & 2.8 & 1.3 & 0.6 & 0.7 & 0.7 & 0.9 & 0.7 & 0.9 & 0.2 & 0.7 \\
\hline [O I] & 6363 & 1.2 & 0.4 & 0.5 & 0.8 & 1.0 & 0.7 & 1.3 & 1.7 & 0.9 & 0.6 \\
\hline [N II $]$ & 6548 & 8.0 & 9.3 & 12.5 & 15.5 & 19.9 & 25.4 & 30.2 & 27.6 & 24.1 & 8.2 \\
\hline $\mathrm{H} \alpha$ & 6563 & 277.8 & 286.0 & 277.2 & 286.0 & 277.4 & 286.0 & 278.3 & 286.0 & 277.7 & 286.0 \\
\hline$[\mathrm{N}$ II $]$ & 6584 & 23.7 & 25.3 & 36.8 & 46.6 & 58.8 & 75.9 & 88.9 & 86.0 & 71.0 & 22.1 \\
\hline He I & 6678 & 1.6 & 2.3 & 1.3 & 2.8 & 2.2 & 2.9 & 2.0 & 0.7 & 2.6 & 3.5 \\
\hline [S II $]$ & 6716 & 2.2 & 0.6 & 3.4 & 0.3 & 1.0 & 1.2 & 6.9 & 7.8 & 0.4 & 0.3 \\
\hline [S II $]$ & 6731 & & & 4.8 & 0.7 & 1.8 & 2.4 & 9.4 & 10.5 & 0.7 & 0.6 \\
\hline He I & 7065 & & & 1.5 & 6.9 & 3.9 & 6.9 & 2.4 & 4.7 & 5.0 & 9.9 \\
\hline [Ar III] & 7136 & & & 1.9 & 5.2 & 5.1 & 6.3 & 6.2 & 4.6 & 5.9 & 5.0 \\
\hline [O II] & 7320 & 26.6 & 33.5 & 10.3 & 27.0 & 20.2 & 19.6 & 8.6 & 10.8 & 11.9 & 15.7 \\
\hline [O II $]$ & 7330 & 14.3 & 24.9 & 8.4 & 21.2 & 10.9 & 14.9 & 7.0 & 8.2 & 6.4 & 12.7 \\
\hline
\end{tabular}

